\title{
VARIAÇÕES MORFOMÉTRICAS EM ABELHAS EUGLOSSINI (HYMENOPTERA: APIDAE) EM RESPOSTA À MUDANÇA FITOFISIONÔMICA
}

\author{
$\underline{\text { Lázaro da Silva Carneiró }}{ }^{1}$; Cândida Maria Lima Aguiar ${ }^{2}$; Willian Moura de Aguiar \\ 1. Bolsista PIBIC/CNPq- AF, Graduando em Bacharelado em Ciências Biológicas, Universidade Estadual de Feira de \\ Santana, e-mail: lazarocarneiro16@gmail.com \\ 2. Orientador, Departamento de Ciências Biológicas, Universidade Estadual de Feira de Santana, e-mail: \\ candida.aguiar@gmail.com \\ 3. Coorientador, Instituto do Meio Ambiente e Recursos Hídricos, e-mail: wmag26@yahoo.com.br
}

PALAVRAS-CHAVE: Abelhas Euglossini; Morfometria; Fenótipo.

\section{INTRODUÇÃO}

As abelhas Euglossini (Hymenoptera: Apidae) são importantes polinizadores de diversas famílias botânicas na região Neotropical, incluindo Orchidaceae, utilizadas pelos machos para coleta de fragrâncias (Dressler, 1982). A maioria das espécies dessas abelhas é solitária, mas diferentes graus de sociabilidade foram reconhecidos em algumas espécies do gênero Euglossa Latreille (Freiria et al. 2017). Estudos realizados com esses insetos tem demonstrado que são potenciais bioindicadores da qualidade do habitat (Aguiar \& Gaglianone 2008, 2012).

Uma maneira de mensurar a resposta dos organismos às variações ambientais é através da morfometria. Essa técnica, baseada no estudo da covariância entre mudanças na forma e fatores casuais (Bookstein, 1991) tem sido usada amplamente em estudos morfológicos focados em abelhas (Nunes et al. 2007, 2008; Ferreira et al. 2012; Francoy et al. 2012).

Contudo, os trabalhos que utilizaram a morfometria geométrica para análises de variações interpopulacionais com abelhas solitárias ainda são escassos, sendo registrados poucos estudos publicados, Ferreira et al. (2011), com Centris aenea Lepeletier, e Neves et al. (2012), com Tetrapedia diversipes Klug. Para abelhas Euglossini, não há estudos relacionados à divergência populacional usando a morfometria. Faz- se necessário entender como populações dessas abelhas respondem, através da morfologia, a diferentes características ambientais. O objetivo desse estudo é avaliar a variabilidade morfológica interpopulacional de machos de uma espécie de abelha Euglossini, Euglossa cordata Linnaeus, proveniente de diferentes fitofisionomias.

\section{MATERIAL E MÉTODOS}

Foram selecionados espécimes de Euglossa cordata que foram coletados em diferentes fitofisiomias (Tabela 1). Os machos foram coletados utilizando armadilhas com iscas aromáticas, conforme descrito em Aguiar \& Gaglianone (2008). Esse material está depositado nas coleções do Laboratório de Estudos Ambientais- LEA e Laboratório de EntomologiaLENT da Universidade Estadual de Feira de Santana-UEFS.

Tabela 1. Número de indivíduos de Euglossa cordata L. analisados por fitofisionomias.

\begin{tabular}{ccccc}
\hline Fitofisionomia & $\begin{array}{c}\text { Município } \\
\text { (BA) }\end{array}$ & Coordenadas & Altitude $(\mathrm{m})$ & $\begin{array}{c}\mathrm{N}^{\circ} \text { de indivíduos } \\
\text { analisados }\end{array}$ \\
\hline CAAR & Pé de Serra & $11^{\circ} 57^{\prime} \mathrm{S} 39^{\circ} 32^{\prime} \mathrm{W}$ & 235 & 45 \\
CERR & Palmeiras & $12^{\circ} 26^{\prime} \mathrm{S} 41^{\circ} 30^{\prime} \mathrm{W}$ & 780 & 73
\end{tabular}




\begin{tabular}{ccccc} 
FESD & Lençóis & $12^{\circ} 29^{\prime} \mathrm{S} 41^{\circ} 21^{\prime} \mathrm{W}$ & 516 & 49 \\
FODM & Ibirapitanga & $13^{\circ} 53^{\prime} \mathrm{S} 39^{\circ} 27^{\prime} \mathrm{W}$ & 660 & 93 \\
FODS & Ibirapitanga & $13^{\circ} 54^{\prime} \mathrm{S} 39^{\circ} 27^{\prime} \mathrm{W}$ & 500 & 94 \\
\hline Total & & & & 354
\end{tabular}

Legenda: CAAR: Caatinga Arbórea; CERR: Cerrado; FESD: Floresta Estacional Semidecídua; FODM: Floresta Ombrófila Densa Montana; FODS: Floresta Ombrófila Densa Submontana.

A asa anterior direita de cada indivíduo foi fixada entre duas lâminas, e fotografada com um estereomicroscópio com câmera acoplada. As fotografias foram levadas para o programa tpsUtil (Rohlf, 2010), criando um banco de imagens que foi aberto no programa tpsDig2 (Rohlf, 2008), onde foram digitalizados 18 marcos anatômicos nas interseções das nervuras das asas (Francoy et al. 2012). Os arquivos foram levados para o software MorphoJ (Klingenberg, 2008) para posterior análise de dados.

Utilizando um paquímetro digital, foi mensurado, em milímetros, o comprimento do mesossoma (CM) e a distância intertegular (DI) de cada indivíduo, representando variáveis de tamanho corpóreo, compondo um banco de dados no programa Excel para análises posteriores.

\section{Análise dos dados}

No Morphoj, foram realizadas as análises de forma e tamanho da asa. Após a superposição de Procrustes, foi aplicado a Análise de Componentes Principais (ACP) e Análise de Variáveis Canônicas (AVC), que permitem observar a variação da forma entre as populações. Para averiguar a similaridade da forma da asa entre cada população, foi usada a distância de Procrustes. No programa PAST (Hammer et al. 2001), foi aplicado um teste de Mantel com o objetivo de averiguar uma correlação entre a distância de Procrustes e a distância geográfica (medida no Google Earth em linha reta).

Para analisar o tamanho da asa, o tamanho do centroide foi extraído do MorphoJ e transferido para PAST, onde foi realizado uma Análise de Variância (ANOVA one way) e o teste a posteriori de Tukey, para observar quais populações diferiram entre si.

Com o objetivo de verificar se há relação entre o tamanho e forma da asa com uma variável ambiental (altitude), foi realizado o teste de Mantel. Para forma, os valores utilizados foram das coordenadas de Procrustes, enquanto para tamanho, o centróide. Essas análises foram feitas no PAST.

Para análise do tamanho corporal (DI e CM), foi aplicada uma análise de variância e seu respectivo teste a posteriori no PAST.

\section{RESULTADOS E DISCUSSÃO}

$\mathrm{Na}$ ACP, foram gerados 32 componentes principais, sendo necessários nove para explicar $71,1 \%$ da variação. A AVC demonstrou que duas das quatro variáveis geradas explicam mais de $80 \%$ da variação. No gráfico de dispersão das duas primeiras variáveis da AVC, não houve separação dos grupos (Figura 1). A maior similaridade da forma da asa, pela distância de Procrustes, foi entre CAAR x FODM, e FODM x FODS (Tabela 2). Ferreira et al. (2011) também observaram que as populações de Centris aenea coletadas em diferentes locais do Nordeste do Brasil não apresentaram variações significativas na forma da asa. Euglossa cordata é indicada como tolerante a diferentes condições ambientais (Aguiar \& Gaglianone, 2012), e assim, é possível que a espécie tenha uma alta plasticidade fenotípica. Não houve correlação entre a distância de Procrustes e a distância geográfica $(r=0.699$, 
$\mathrm{p}=0.084$ ), o que pode está relacionado à elevada capacidade de dispersão de Euglossa cordata.

O tamanho da asa apresentou diferenças significativas entre as populações (ANOVA one way $\mathrm{f}=10.58, \mathrm{p}=0.000$ ), e o teste de Tukey indicou que as variações ocorreram entre CERR x FESD, FODS x CAAR; FODS x FESD; FODM x CAAR; FODM x FESD. Isso pode ser reflexo das diferentes características fitofisionômicas, que envolveriam a disponibilidade de recursos, e pressões antrópicas relacionadas à fragmentação, principalmente na Caatinga e Mata Atlântica.

$\mathrm{O}$ teste de Mantel indicou correlação positiva entre a altitude e a forma das asas ( $\mathrm{r}=0.036, \mathrm{p}=0.038)$, mas não com o tamanho das asas $(\mathrm{r}=-0.034, \mathrm{p}=0.88)$. O complexo de serras da Chapada Diamantina pode estar agindo como uma barreira entre as populações, levando a uma diferenciação na forma da asa, resultado semelhante ao observado por Nunes et al. (2007) em Melipona scutelaris Lepeletier, .

Para o tamanho corpóreo, a DI não apresentou diferenças entre as populações (ANOVA one way $\mathrm{f}=1.28, \mathrm{p}=0.273$ ), contudo o $\mathrm{CM}$ indicou variações (Kruskal-Wallis $\mathrm{H}=11.5, \mathrm{p}=0.02$ ), e o teste a posteriori de Mann-Whitney indicou que essas variações ocorreram entre as populações de FESD, FODM e FODS, com CERR e CAAR. Isso pode ser reflexo nas diferenças na disponibilidade de recursos, já que em abelhas o tamanho corpóreo está ligado à obtenção de alimento (Peruquetti, 2003).

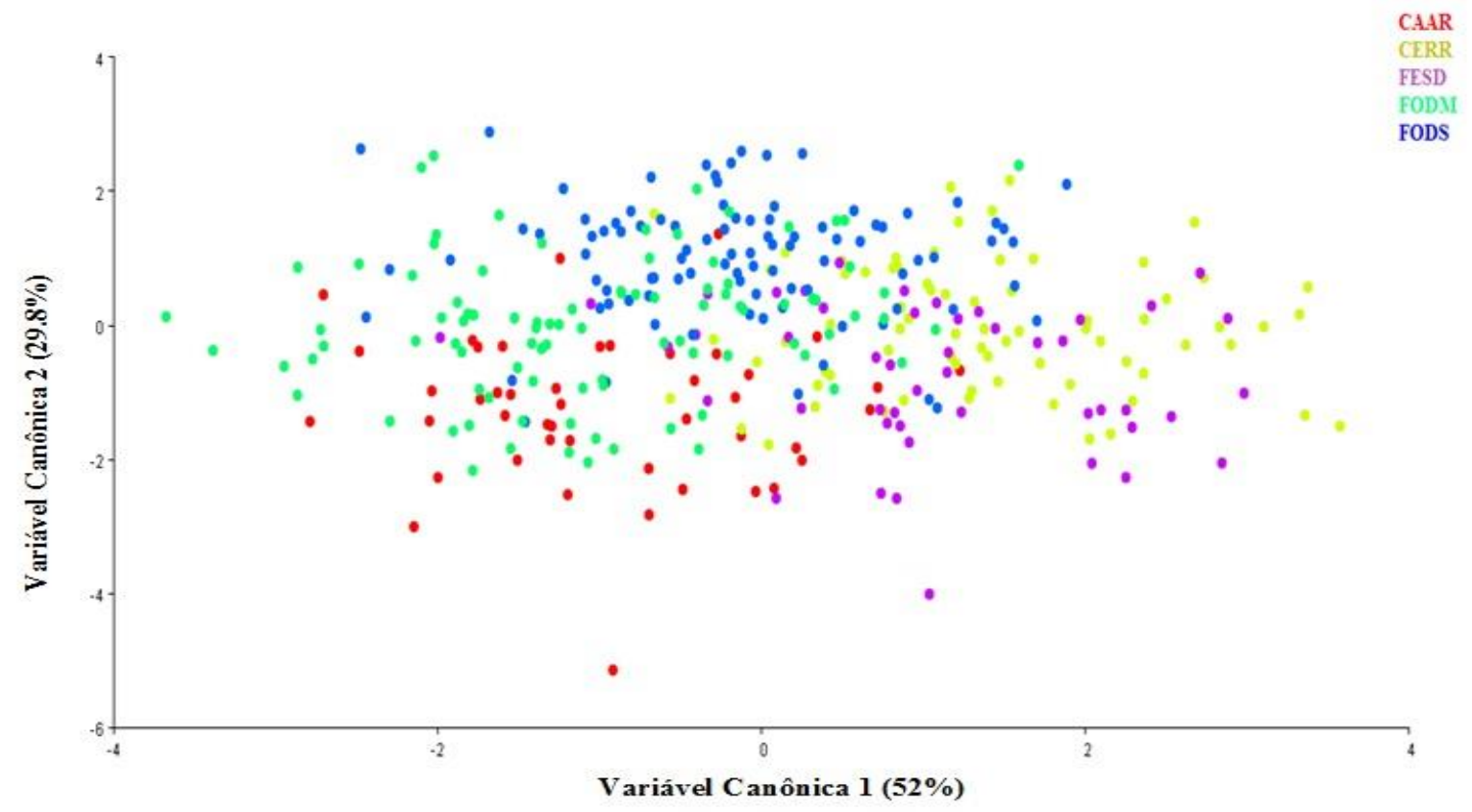

Figura 1. Dispersão das cinco populações de Euglossa cordata L. gerado a partir das duas variáveis canônicas com maior variação.

Tabela 2. Distância de Procrustes gerada a partir da Análise de Variáveis Canônicas (AVC) entre cada fitofisionomia.

\begin{tabular}{ccccc}
\hline & CAAR & CERR & FODM & FESD \\
\hline CERR & 0,0105 & & & \\
FODM & 0,0054 & 0,0095 & & \\
FODS & 0,0081 & 0,0079 & 0,0054 & \\
FESD & 0,0082 & 0,0078 & 0,0093 & 0,0081 \\
\hline
\end{tabular}




\section{CONSIDERAÇÕES FINAIS}

Euglossa cordata parece apresentar uma plasticidade fenotípica referente à forma da asa, enquanto que para o tamanho da asa, as variações ambientais parecem levar a mudanças fenotípicas entre as populações. Para o tamanho corpóreo, as variáveis responderam diferentemente, sendo o comprimento do mesossoma mais sensível para detectar variações morfométricas.

\section{REFERÊNCIAS}

AGUIAR, W. M. D.; \& GAGLIANONE, M. C. 2012. Euglossine bee communities in small forest fragments of the Atlantic Forest, Rio de Janeiro state, southeastern Brazil (Hymenoptera, Apidae). Rev. Bras. de Entomol. 56(2): 210-219.

AGUIAR, W. M.; \& GAGLIANONE, M. C. 2008. Comunidade de abelhas Euglossina (Hymenoptera: Apidae) em remanescentes de mata estacional semidecidual sobre tabuleiro no estado do Rio de Janeiro. Neotrop Entomol. 37(2): 118-125.

BOOKSTEIN, F. L. 1991. Morphometric tools for landmark data. Geometry and Biology. Cambridge University Press: New York.

CAMERON, S. A. 2004. Phylogeny and biology of neotropical orchid bees (Euglossini). Annu. Rev. Entomol. 49(1): 377-404.

DRESSLER, R. L.1982. Biology of the orchid bees (Euglossini). Annu. Rev. Ecol. Evol. Syst. 13: 373-394.

FERREIRA, V. S.; AGUIAR, C. M. L.; COSTA, M. A.; SILVA, J. G. 2011. Morphometric Analysis of Populations of Centris aenea Lepeletier (Hymenoptera: Apidae) from Northeastern Brazil. Neotrop Entomol. 40(1): 97-102.

FRANCOY, T. M.; DE FARIA FRANCO, F.; \& ROUBIK, D. W. 2012. Integrated landmark and outline-based morphometric methods efficiently distinguish species of Euglossa (Hymenoptera, Apidae, Euglossini). Apidologie 43(6): 609-617.

FREIRIA, G. A.; GARÓFALO, C. A.; \& DEL LAMA, M. A. 2017. The primitively social behavior of Euglossa cordata (Hymenoptera, Apidae, Euglossini): a view from the perspective of kin selection theory and models of reproductive skew. Apidologie: 1-10.

HAMMER, O.; HARPER, D. A. T.; \& RYAN, P. D. 2001. PAST-Palaeontological Statistics, ver. 1.89. Palaeontologia electronica 4(9). Disponível em: http://palaeoelectronica.org/2001_1/past/past.pdf/Acessado em 19 de julho de 2017

KLINGENBERG, C. P. 2008. MorphoJ. Faculty of Life Sciences, Universityof Manchester 3: 7577.

NEVES, C. M. L.; CARVAlHO, C. A. L.; SOUZA, A. V.; \& JUNIOR, C. A. L. 2012. Morphometric Characterization of a Population of Tetrapedia diversipes in Restricted Areas in Bahia, Brazil (Hymenoptera: Apidae). Sociobiology 59(3): 767-782.

NUNES, L. A.; ARAUJO, E. D.; CARVALHO, C. A. L.; \& WALDSCHMIDT, A. M. 2008. Population divergence of Melipona quadrifasciata anthidioides (Hymenoptera: Apidae) endemic to the semiarid region of State of Bahia, Brazil. Sociobiology 52(1): 81-93.

NUNES, L. A.; PINTO, M. F. F. C.; CARNEIRO, P.; PEREIRE, D. G.; \& WALDSCHMIDT, A. M. 2007. Divergência Genética em Melipona scutellaris Latreille (Hymenoptera: Apidae) com base em Caracteres Morfológicos. Biosci. J. 23(1): 1-9.

PERUQUETTI, R. C.2003. Variação do tamanho corporal de machos de Eulaema nigrita Lepeletier (Hymenoptera, Apidae, Euglossini). Resposta materna à flutuação de recursos? Rev. Bras. Zool. 20: 207- 212.

ROHLF, F. J. 2008. TpsDIG2. 12. Department of Ecology and Evolution, State University of New York at Stony Brook.

ROHLF, F. J. 2010. tpsUtil version 1.44. Department of Ecology and Evolution, State University of New York at Stony Brook. 